\title{
BMJ Open Effect of educational interventions on knowledge of the disease and glycaemic control in patients with type 2 diabetes mellitus: a systematic review and meta- analysis of randomised controlled trials
}

Wondimeneh Shibabaw Shiferaw (D) , ${ }^{1}$ Tadesse Yirga Akalu (D) , ${ }^{2}$ Melaku Desta, ${ }^{3}$ Ayelign Mengesha Kassie (D) , ${ }^{4}$ Pammla Margaret Petrucka, ${ }^{5}$ Yared Asmare Aynalem (iD ${ }^{6}$

To cite: Shiferaw WS, Akalu TY, Desta M, et al. Effect of educational interventions on knowledge of the disease and glycaemic control in patients with type 2 diabetes mellitus: a systematic review and meta-analysis of randomised controlled trials. BMJ Open 2021;11:e049806. doi:10.1136/ bmjopen-2021-049806

- Prepublication history for this paper is available online To view these files, please visit the journal online (http://dx.doi. org/10.1136/bmjopen-2021 049806).

Received 02 February 2021 Accepted 29 0ctober 2021
D) Check for updates

(c) Author(s) (or their employer(s)) 2021. Re-use permitted under CC BY-NC. No commercial re-use. See rights and permissions. Published by BMJ.

For numbered affiliations see end of article.

Correspondence to Wondimeneh Shibabaw Shiferaw;

wshibabaw2015@gmail.com

\section{ABSTRACT}

Background Globally, type 2 diabetes has continued to increase, now accounting for over $90 \%$ of all diabetes cases. Though the magnitude of uncontrolled glycaemic levels in patients with type 2 diabetes is steadily rising, evidence showed that effectively controlled glycaemic levels can prevent complications and improve the quality of life of these patients. As little is known about the effect of educational interventions on this population, this systematic review and meta-analysis evaluated the effectiveness of educational interventions versus standard care on glycaemic control and disease knowledge among patients with type 2 diabetes.

Methods PubMed, Google Scholar, Cochrane Library, Scopus, African Journals Online and Wiley Online Library were searched. Two authors independently assessed within-trial risk of bias in each included study using revised Cochrane risk-of-bias tool for randomised trials. A random-effects model was employed to estimate combined effect sizes. Subgroup analyses were employed to investigate possible sources of heterogeneity between studies. The overall certainty of the evidence was evaluated using the Grading of Recommendations Assessment, Development and Evaluation approach.

Results A total of 19 trials with 2708 study participants were included in the review. Primary outcomes (glycaemic control) were reported in 18 trials. The pooled estimated impact of educational intervention on glycaemic levels using the random-effects model was $-0.83(95 \% \mathrm{Cl}$ : -1.17 to $-0.49, p<0.001)$. Subgroup analyses revealed greater $\mathrm{A} 1 \mathrm{c}$ reductions in those studies with intervention duration of up to 3 months and with empirical intervention designs. Educational interventions led to significant increases in participants' knowledge of type 2 diabetes (standardised mean difference: 1.16 ; $95 \% \mathrm{Cl}: 0.71$ to 1.60 ; $\mathrm{I}^{2}=93 \%$ ).

Conclusion In the current review overall, educational interventions can potentially lead to improved glycaemic control levels in patients with type 2 diabetes despite heterogeneity across the studies. Besides, the findings showed that educational interventions could increase disease knowledge among patients with type 2 diabetes.
Strengths and limitations of this study

- This systematic review will provide a comprehensive search of the literature, the effect of educational intervention on glycaemic control and knowledge of type 2 diabetes.

- An extensive search of multiple databases and search engines (ie, PubMed, African Journals Online, Web of Science, Scopus and Google Scholar) was performed to ensure a comprehensive review; nevertheless, potentially relevant articles from other/ additional databases may be missed.

- We only used English-language articles, although our target was global, which could be in several other languages such as Spanish, French or Portuguese.

PROSPERO registration number CRD42020205838.

\section{BACKGROUND}

Diabetes mellitus (DM) is increasingly becoming an extensive non-communicable health problem, leading to significant morbidity and mortality. ${ }^{1}$ Globally, a recent estimate showed that approximately 422 million adults are living with DM. $^{2}$ According to the International Diabetes Federation projection, approximately 629 million people will be affected by $2045 .^{3}$ Of these, approximately $80 \%$ of affected individuals live in low-income countries. ${ }^{4}$ In particular, type $2 \mathrm{DM}$ (T2DM) is responsible for more than $90 \%$ of all diabetes cases. ${ }^{5}$ The increasing burden is due to several risk factors such as sedentary behaviours, obesity, unhealthy diet, lack of exercise, family history and age. ${ }^{6-8}$

Maintaining optimal glycaemic levels is vital to diabetes control. ${ }^{9}$ However, evidence showed that poor glycaemic control 
(glycosylated haemoglobin $(\mathrm{HbA1c}) \geq 7 \%$ ) contributes to kidney failure, myocardial infarction, stroke, retinopathy, hypertension, increasing costs for patient care and reduced quality of life. ${ }^{10-14}$ The aims of T2DM management are to attain glycaemic targets, minimise adverse events and prevent complications. ${ }^{15} 16$ Therefore, lifestyle modifications, such as diet and exercise, have been reported to reduce the complications of uncontrolled glycaemic levels in patients with T2DM. ${ }^{17}$

Evidence has revealed that self-management education can reduce the glycaemic level by $30 \%-80 \% .{ }^{18}$ Besides, diabetes education can improve glycaemic control, change people's behaviours, promote self-care, and reduce complications and progression of the disease. ${ }^{19-21}$ Moreover, numerous studies suggest that diabetes educational interventions can increase knowledge of diabetes $^{22}{ }^{23}$ and medication compliance, ${ }^{24}$ decrease readmissions, ${ }^{25}{ }^{26}$ reduce length of stay and mortality rate, ${ }^{25}$ and improve glycaemic control. ${ }^{27}{ }^{28}$ In addition, the American Diabetes Association (ADA) position statement provides the evidence and strategies for the provision of education and support services to all adults living with T2DM. ${ }^{29}$ Moreover, the consensus report showed that there are four critical times to provide diabetes selfmanagement education and support: (1) at diagnosis, (2) annually and/or when not meeting treatment targets, (3) when complicating factors develop, and (4) when transitions in life and care occur. ${ }^{30}$

Though knowledge about diabetes has paramount benefit to patients' self-care management, insufficient diabetes knowledge is unfavourable to the patients' health due to most of the complications that arise can be prevented through self-care practice. ${ }^{31} 32$ However, numerous studies have shown that improving patient knowledge about T2DM and its complications has substantial benefits to maintain optimal glycaemic levels, enhance treatment adherence, reduce treatment cost and decrease the progression of disease. ${ }^{33-35}$ Previously, several reviews on the effect of self-management interventions for patients with T2DM exist. ${ }^{36-38}$ However, most reviews included inadequate number of articles, did not address the effects of education on knowledge of diabetes and included interventions which were poorly described. Thus, research is required to estimate the effects of educational interventions on sufficient methodological quality and substantive statistical analysis. Hence, the present review and meta-analysis aimed to evaluate the effect of educational interventions on glycaemic control and disease knowledge in patients with T2DM.

\section{Review questions}

- Does a structured educational intervention increase diabetes knowledge in patients with T2DM?

- Does a structured diabetes educational intervention reduce HbA1c levels among patients with T2DM?
METHODS

\section{Protocol and registration}

Initially, PROSPERO was searched to confirm for other reviews on the effect of educational interventions on glycaemic control and disease knowledge among patients with T2DM. But no such reviews were identified. Thus, the protocol was registered on PROSPERO (www.crd.york. ac.uk/PROSPERO/) as recommended by the Preferred Reporting Items for Systematic Review and Meta-Analysis (PRISMA) statement. ${ }^{39}$

\section{Search strategy and data sources}

We did a comprehensive systematic search to collect all relevant articles using the Peer Review of Electronic Search Strategies for systematic reviews. ${ }^{40}$ The search was limited to studies published in peer-reviewed journals from January 2000 to August 2021 (as authors were interested in up-to-date data). The PRISMA guidelines were used to conduct and report the present review. ${ }^{39}$ The literature was searched in PubMed, Scopus, Google Scholar, African Journals Online, Cochrane Library and Wiley Online Library. The keywords used for the review included "educational", "behavioral", "knowledge", "glycemic control", "glycosylated hemoglobin", "HbAlc", "Type 2 diabetes mellitus", "Type 2 diabetes" and "T2DM". Boolean operators like 'AND' and 'OR' were used to combine search terms. The Medical Subject Headings $(\mathrm{MeSH})$ terms employed in the PubMed search engine in various combinations are shown in table 1 . To access all articles on this topic, we manually review all references to reduce publication bias. Searches were performed on 20 August 2020. The search was restricted to full texts, human studies and English-language publications. In the present review, the Patient/Population $(\mathrm{P})$; Intervention (I); Comparison (C) and Outcomes (O) question was as follows: is educational intervention (I) in people with T2DM (P), when compared with people who had not taken part in the educational intervention or had standard care $(\mathrm{C})$, associated with improved glycaemic control and disease knowledge $(\mathrm{O})$ ?

\section{Eligibility criteria}

Types of participants

This review takes into consideration studies that included adult patients ( $\geq 18$ years old) with T2DM in outpatient health settings, primary care settings, diabetes clinics and hospitals within the catchment. Those articles focusing on or including children or those with T1DM were excluded from the review.

\section{Types of interventions}

The review considered any educational intervention provided to adult patients with T2DM in diabetes care settings receiving standard or routine care. Intervention could be provided by any healthcare provider, involved any medium (written, oral, video and computer), delivered at the individual or group level, focused on theorybased or empirical content, and of varying duration. 


\begin{tabular}{llc}
\hline Table 1 & PubMed search history & \\
\hline Search & Search terms & Hits \\
\hline$\# 1$ & $\begin{array}{l}\text { Type } 2 \text { diabetes[tw] OR Type } 2 \text { diabetes mellitus[tw] OR T2DM[tw] OR insulin non dependent } \\
\text { diabetes [tw] }\end{array}$ & 199276 \\
$\# 2$ & Education [tw] OR intervention [tw] OR behavioral intervention[tw] OR self-management [tw] & 1587693 \\
$\# 3$ & Glycemic control [tw] OR glycosylated hemoglobin[tw] OR HbA1c[tw] \\
$\# 4$ & Knowledge [tw] OR behavioral outcomes [tw] & 65114 \\
$\# 5$ & \#1 AND \#2 AND \#3 AND \#4 \\
$\# 6$ & \#5; limits: studies done with humans, English language, full text, RCT and publication year
\end{tabular}

$\mathrm{RCT}$, randomised controlled trial.

Studies lacking an education intervention, unclear information respecting the intervention and insufficient data on the main outcome variable were considered criteria for exclusion.

The comparator in this study was the delivery of the usual care/routine care for T2DM. Routine care refers to diabetes care that healthcare staff usually and normally provides in their daily care. Articles were excluded if they did not implement a comparison with routine care.

\section{Types of studies}

In the present review, studies were included if they were randomised controlled trials. Full-text articles were included, whereas studies published with only abstract or unpublished data were excluded. Of note, nonrandomised controlled trials, quasi-experimental, before and after, cohort, case-control and cross-sectional studies were excluded because uncontrolled trials and observational studies lead to greater risk of biased estimates of effect size. ${ }^{41}$

\section{Type of outcome}

This review included the following outcome measures: glycaemic control as the primary outcome of the metaanalysis, and knowledge of diabetes considered as the secondary outcome. A study was excluded if outcomes were not measured or data could not be extracted.

\section{Study selection}

After database exploration, all recognised studies were uploaded into EndNote V.8, and duplicate articles were removed. Predefined selection criteria were used to select relevant full-text articles during the screening process. Three authors (WSS, PMP and YAA) independently screened the title, abstract and keywords of the studies identified for possible eligibility in the review. Afterward, all full-text articles were evaluated carefully for inclusion and data extraction. Further screening of full text was done by two (TY and YAA) independent authors to select the studies which satisfied the eligibility standards. The possible justification for the exclusion of full-text studies was documented and reported in the systematic review. Any uncertainties about study eligibility were discussed between authors.

\section{Data extraction}

After identifying studies for eligibility, data abstraction was conducted by two (AMK and WSS) independent authors using Microsoft Excel (version 10) for Windows. The first author undertook the data abstraction, whereas the second author assumed control for the quality of extracted and entered data. The data extracted from each study included first/corresponding author, year of publication, study setting, education provider, duration of intervention, intensity of intervention, components of the intervention, number of participants in each arm (intervention and standard care group), intervention design, outcome measures, before and after intervention HbAlc levels, and knowledge scores. The outcome measures in this review were reported as the variation from starting point to closing date of follow-up in the intervention and standard care groups. If the SD of mean difference (MD) was not reported in an included study, the values were recalculated according to the guideline in the Cochrane Handbook. ${ }^{42}$

Assessment of risk of bias in included studies

Two (MD and PMP) independent authors assessed withintrial risk of bias in each included study using revised Cochrane risk-of-bias tool for randomised trials (RoB 2). ${ }^{43}$ The Cochrane's RoB 2 tool evaluation domains used to evaluate validity and bias in studies of clinical trials were applied regarding randomisation, allocation sequence concealment, blinding, incomplete outcome data, selective outcome reporting and other biases. For this review, the overall risk of bias was rated as high/ low/some concerns, in agreement with the RoB 2 tool. Any disagreement was resolved through discussion and consensus.

\section{Assessment of certainty of the evidence}

To evaluate the quality of the evidence, the authors used the Grading of Recommendations Assessment, Development and Evaluation (GRADE) approach. ${ }^{44}$ GRADE proGDT was employed to summarise the quality of evidence. ${ }^{45}$ The certainty of the evidence encompasses consideration of the within-study risk of bias which comprises methodological worth, indirectness of evidence, unexplained 
heterogeneity, imprecision and probability of publication bias. The GRADE approach has four levels of quality such as high-quality evidence that recommends that additional study is very unlikely to change our confidence in the estimate of effect size; moderate quality reflects further research as likely to have a vital impact on the estimate of effect size and may alter the estimate; low quality reveals that further research is very unlikely to have a significant influence on the current estimate of effect size and is likely to change the estimate; and very low quality suggests one is precise indeterminate about the estimate.

\section{Data synthesis and analysis}

The primary and secondary outcomes were reported as MD and standardised MD (SMD) with a 95\% CI, using a random-effects model, ${ }^{46}$ respectively. Degree of heterogeneity was examined with the $\mathrm{I}^{2}$ statistic, which expresses the amount of heterogeneity between studies. ${ }^{47}$ To interpret the effect sizes, authors followed Cohen's guidelines where $\mathrm{d} \leq 0.2$ was small, $\mathrm{d} \approx 0.5$ was medium and $\mathrm{d}>0.8$ were large variation among intervention and control groups. ${ }^{48}$ We performed subgroup analyses to reduce the level of heterogeneity for the primary outcomes using duration of intervention and intervention design. Publication bias was visually evaluated using the funnel plot, supplemented by Egger's regression test. ${ }^{49}{ }^{50}$ Sensitivity analysis was performed due to the high degree of heterogeneity and risk of bias. Review Manager of the Cochrane Collaboration (RevMan V.5.4, Cochrane Organization) was used to perform the meta-analysis.

\section{Patient and public involvement}

Patients and/or the public were not involved in the design, or conduct, or reporting, or dissemination plans of this research.

\section{RESULTS}

\section{Selection of studies}

The search of the six databases yielded 1183 articles, and 4 articles were retrieved manually through a review of reference lists. After eliminating duplicates, 457 articles remained. Three hundred seventy-two studies were removed after reading the abstract and title, leaving 85 articles for full screening. Following 66 exclusions at the full-text level (mainly due to non-randomised controlled trials $(n=34)$, or reporting mixed population (type 1 and type 2 diabetes) $(\mathrm{N}=7)$ ), 19 studies were incorporated in the final review. The flow diagram for study selection is shown in figure 1 .

\section{Characteristics of the included studies}

In the current meta-analysis, a total of 19 articles met the inclusion criteria with 2708 study participants. Of these, nine included articles reported glycaemic control and knowledge of diabetes as a common outcome variable. Regarding location, two were from Brazil, ${ }^{51}{ }^{52}$ three from China, ${ }^{53-55}$ two from Germany, ${ }^{567}$ five from Iran, ${ }^{58-62}$ two

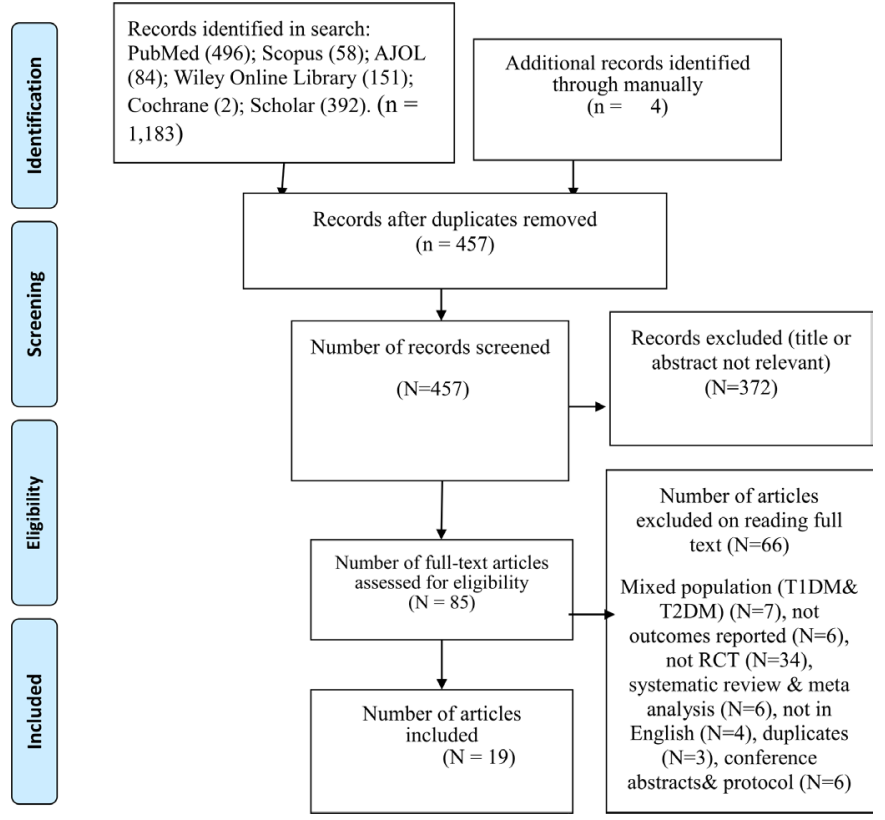

Figure 1 PRISMA flow chart for selection of studies. PRISMA, Preferred Reporting Items for Systematic Review and Meta-Analysis; RCT, randomised controlled trial; T1DM, type 1 diabetes mellitus; T2DM, type 2 diabetes mellitus.

from Malaysia, ${ }^{63}{ }^{64}$ two from Sweden, ${ }^{65} 66$ and one each from Thailand, ${ }^{67}$ Sri Lanka, ${ }^{68}$ and Australia. ${ }^{69}$ The sample size varied from $60^{53}$ to 300 participants. ${ }^{55}$ Educational interventions in the review were guided by the following theories or models: three studies used the theory of selfefficacy, ${ }^{546367}$ three studies ${ }^{566166}$ used empowerment theory, two studies ${ }^{60} 68$ used theory of self- efficacy and motivational interviewing, and one study used either chronic care model, ${ }^{55}$ PRECEDE-PROCEED model, ${ }^{62}$ BASNEF model ${ }^{59}$ or behavioural theory. ${ }^{64}$ However, the remainder of the articles ${ }^{51-5357586569}$ used non-theory or model-based approaches.

The educational interventions comprised of faceto-face counselling, ${ }^{54} \quad 55636869$ diabetes education sessions, ${ }^{515255606367}$ group discussion, ${ }^{5658-6067}$ telephone follow-ups, ${ }^{5460636769}$ home visits, ${ }^{67}$ demonstrations, ${ }^{53} 5459$ as well as questions and responses ${ }^{589}$ as among the most common approaches. The control groups of all studies were the current standard of care. The duration of educational interventions varied from 4 weeks ${ }^{54} 58$ to 12 months. ${ }^{52}{ }^{66}$ Intervention groups obtained the information by different healthcare professionals such as physicians, ${ }^{545566}$ nurses,${ }^{5256586769}$ nutritionists, ${ }^{6164}$ health managers, ${ }^{55}$ public health assistants ${ }^{55}$ and pharmacists. ${ }^{51}$ In most included studies, intervention processes were group-based education ${ }^{52}$ 54-63 65-67 and combined education $^{536869}$; however, in the remainder, web-based ${ }^{64}$ and individual-based $^{51}$ education approaches were used. The main results and features of the selected studies are presented in table 2.

\section{Risk of bias in the included studies}

The random sequence generation for allocation was evaluated as low risk of bias in 12 studies, ${ }^{52-5456576061636466-68}$ 


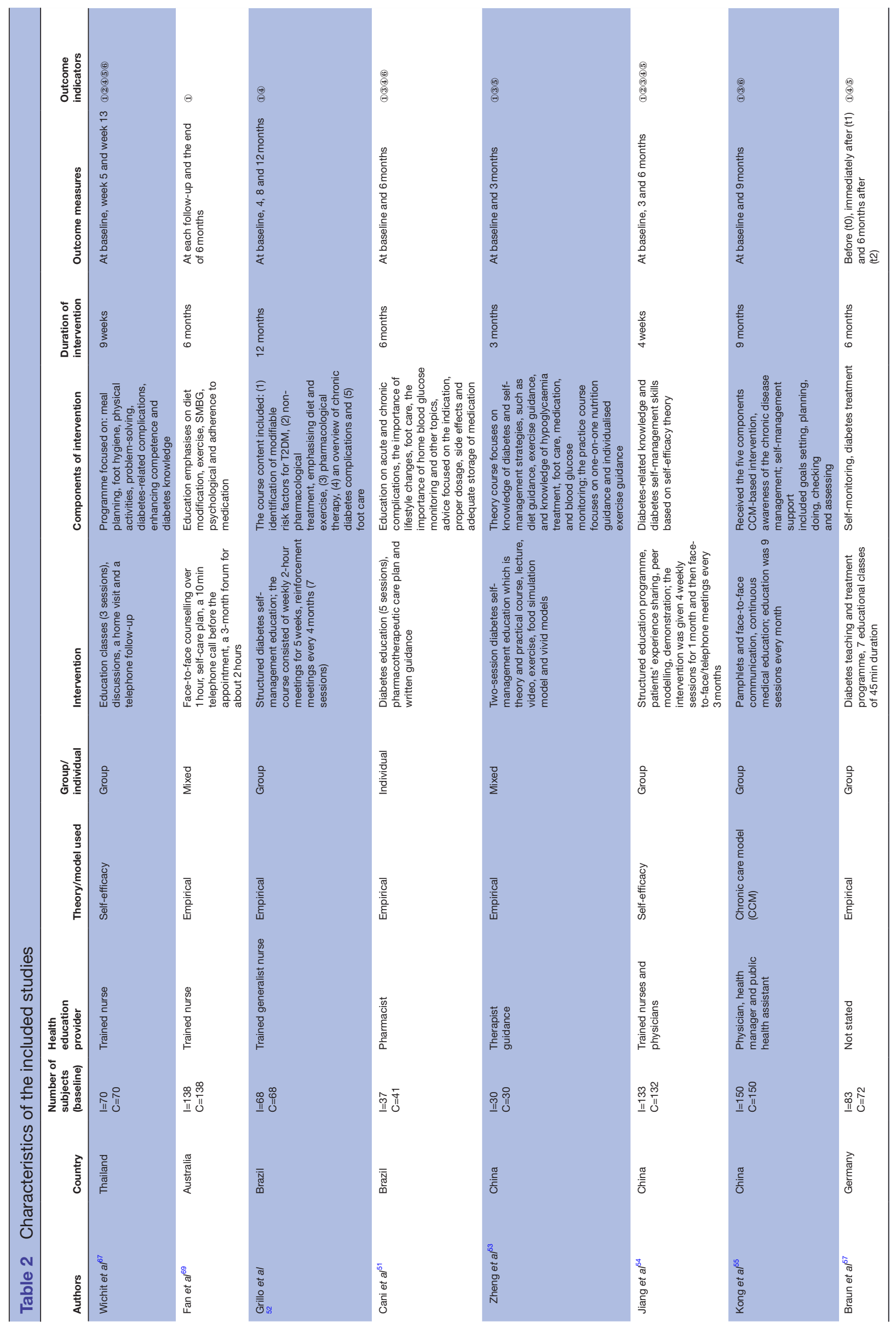

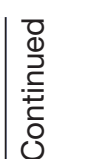



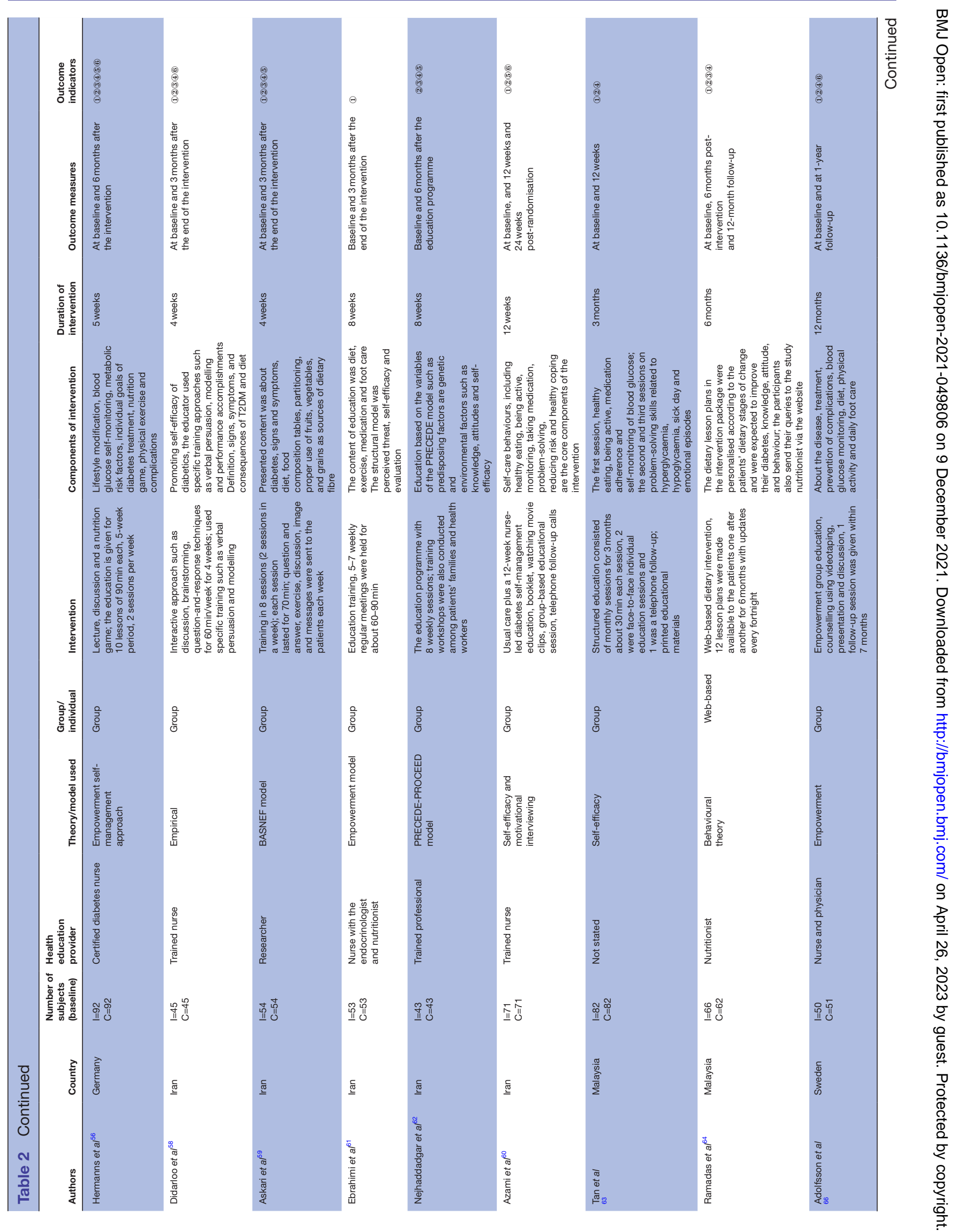

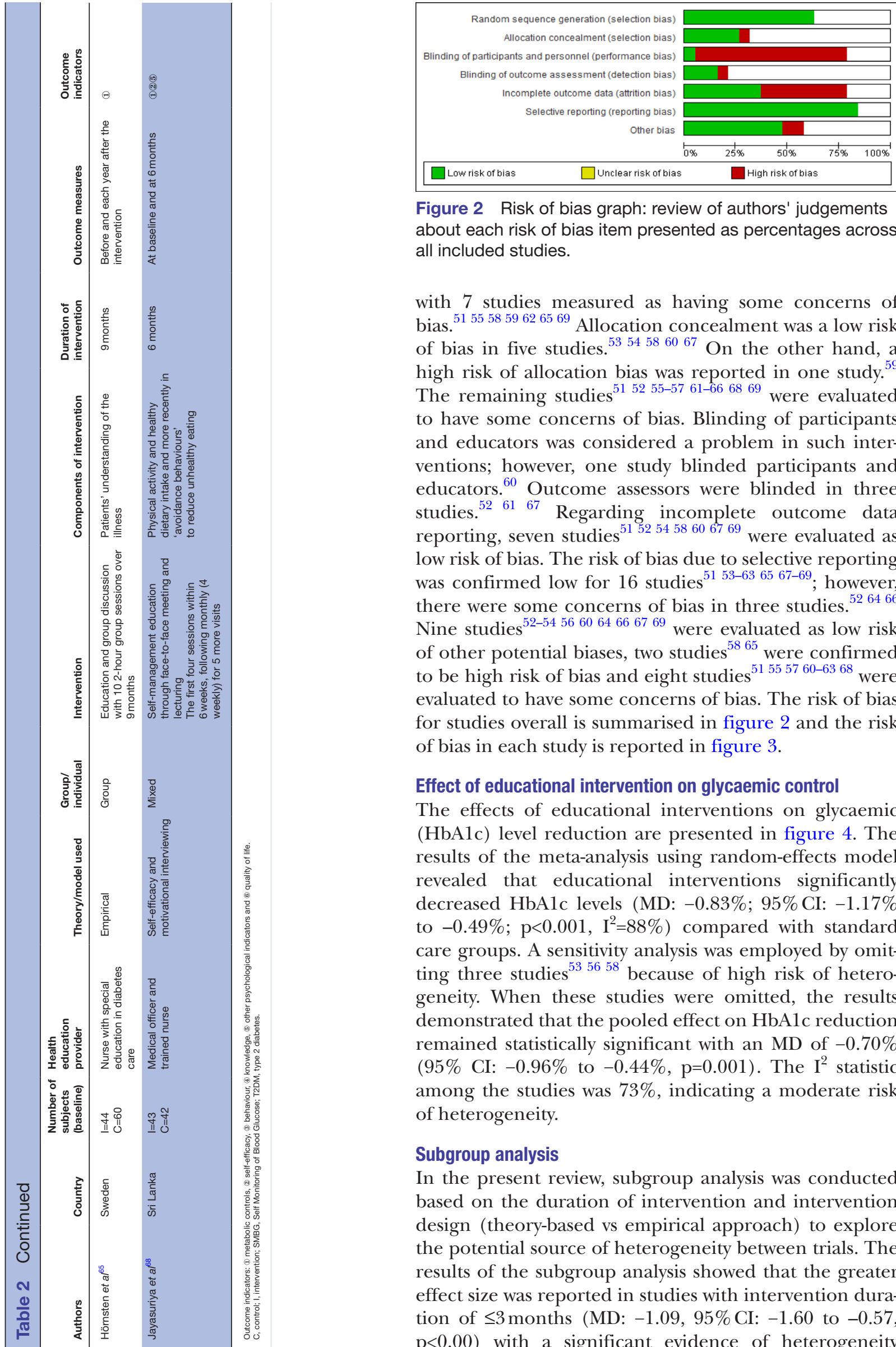

Figure 2 Risk of bias graph: review of authors' judgements about each risk of bias item presented as percentages across all included studies.

with 7 studies measured as having some concerns of bias. ${ }^{51} 555859626569$ Allocation concealment was a low risk of bias in five studies. ${ }^{5354586067}$ On the other hand, a high risk of allocation bias was reported in one study. ${ }^{59}$ The remaining studies ${ }^{51} 52$ 55-57 61-66 6869 were evaluated to have some concerns of bias. Blinding of participants and educators was considered a problem in such interventions; however, one study blinded participants and educators. ${ }^{60}$ Outcome assessors were blinded in three studies. $^{52} 6167$ Regarding incomplete outcome data reporting, seven studies ${ }^{51525458606769}$ were evaluated as low risk of bias. The risk of bias due to selective reporting

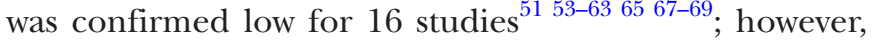
there were some concerns of bias in three studies. ${ }^{526466}$ Nine studies ${ }^{52-54} 566064666769$ were evaluated as low risk of other potential biases, two studies ${ }^{58} 65$ were confirmed to be high risk of bias and eight studies ${ }^{51555760-6368}$ were evaluated to have some concerns of bias. The risk of bias for studies overall is summarised in figure 2 and the risk of bias in each study is reported in figure 3 .

\section{Effect of educational intervention on glycaemic control}

The effects of educational interventions on glycaemic (HbAlc) level reduction are presented in figure 4 . The results of the meta-analysis using random-effects model revealed that educational interventions significantly decreased HbA1c levels (MD: $-0.83 \%$; 95\% CI: $-1.17 \%$ to $-0.49 \% ; \mathrm{p}<0.001, \mathrm{I}^{2}=88 \%$ ) compared with standard care groups. A sensitivity analysis was employed by omitting three studies ${ }^{53} 5658$ because of high risk of heterogeneity. When these studies were omitted, the results demonstrated that the pooled effect on $\mathrm{HbAlc}$ reduction remained statistically significant with an MD of $-0.70 \%$ (95\% CI: $-0.96 \%$ to $-0.44 \%, \mathrm{p}=0.001)$. The $\mathrm{I}^{2}$ statistic among the studies was $73 \%$, indicating a moderate risk of heterogeneity.

\section{Subgroup analysis}

In the present review, subgroup analysis was conducted based on the duration of intervention and intervention design (theory-based vs empirical approach) to explore the potential source of heterogeneity between trials. The results of the subgroup analysis showed that the greater effect size was reported in studies with intervention duration of $\leq 3$ months (MD: -1.09 , 95\% CI: -1.60 to -0.57 , $\mathrm{p}<0.00$ ) with a significant evidence of heterogeneity 


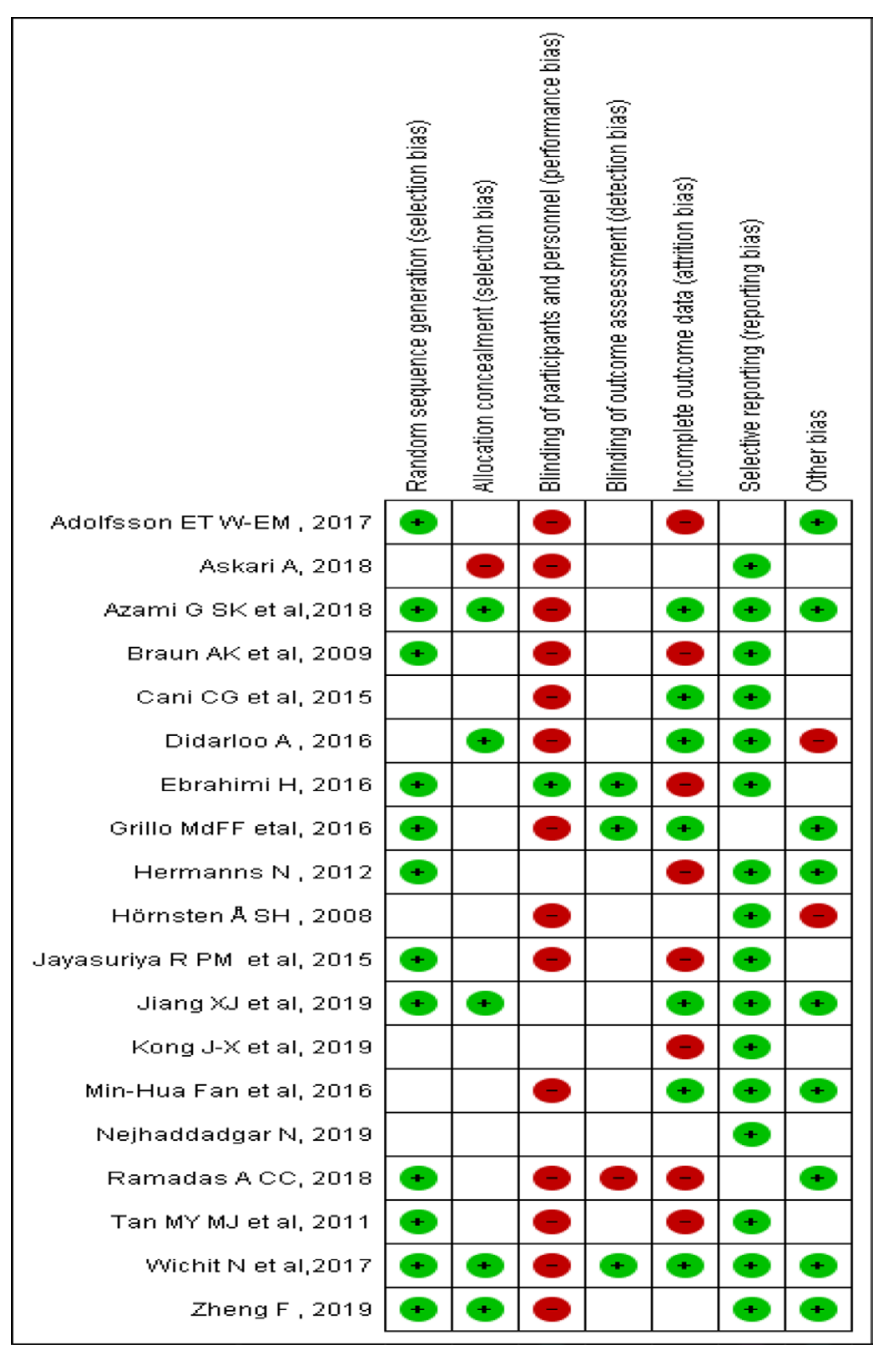

Figure 3 Risk of bias summary: review of authors' judgements about each risk of bias item for each included study.

among studies $\left(I^{2}=88 \%\right)$ (figure 5$)$. Additionally, the results of the subgroup analysis revealed that interventions with an empirical approach had greater effects in terms of reducing glycaemic levels (MD: $-1.03,95 \% \mathrm{CI}$ : -1.90 to $-0.15, \mathrm{p}<0.00)$. Because of a significant degree of heterogeneity between studies $\left(\mathrm{I}^{2}=88 \%\right)$, a randomeffects analysis was used (figure 6).

The effect of educational interventions on diabetes knowledge Ten out of the 19 studies reported knowledge of diabetes as an outcome variable. ${ }^{51} 5254$ 56-5962 6467 The pooled effect size of the 10 trials demonstrated an improvement in knowledge of T2DM (SMD: 1.16, 95\% CI: 0.7 to 1.60, $\mathrm{p}<0.001$; figure 7) compared with standard care groups. A random-effects model was used because of significant heterogeneity. The Dietary Knowledge Questionnaire ${ }^{64}$ and the Diabetes and Medication Knowledge Questionnaire ${ }^{5254575867}$ were used to estimate the level of knowledge in individuals with T2DM. The number of items was between $8^{62}$ and 24 items. ${ }^{67}$ There was a significant variation in knowledge of T2DM scores across different studies.

\section{Publication bias}

The presence of publication bias was visually evaluated using a funnel plot for the primary outcome (glycaemic control), and the results also reported there was no publication bias (figure 8). Likewise, Egger's test also showed no publication bias $(\mathrm{p}=0.732)$. On the other hand, there were insufficient data to generate funnel plots to assess for the potential presence of publication bias for the second outcome (knowledge about T2DM).

\section{Overall quality of the evidence}

The overall quality of evidence was assessed using the GRADE approach and the results are presented in the summary of findings for the main comparison. Findings showed that the overall certainty of the evidence for glycaemic control was moderate, which suggests further studies will increase our confidence in the estimate of effect size. The quality of evidence for diabetes knowledge was low, which reflects that the effect size is limited 


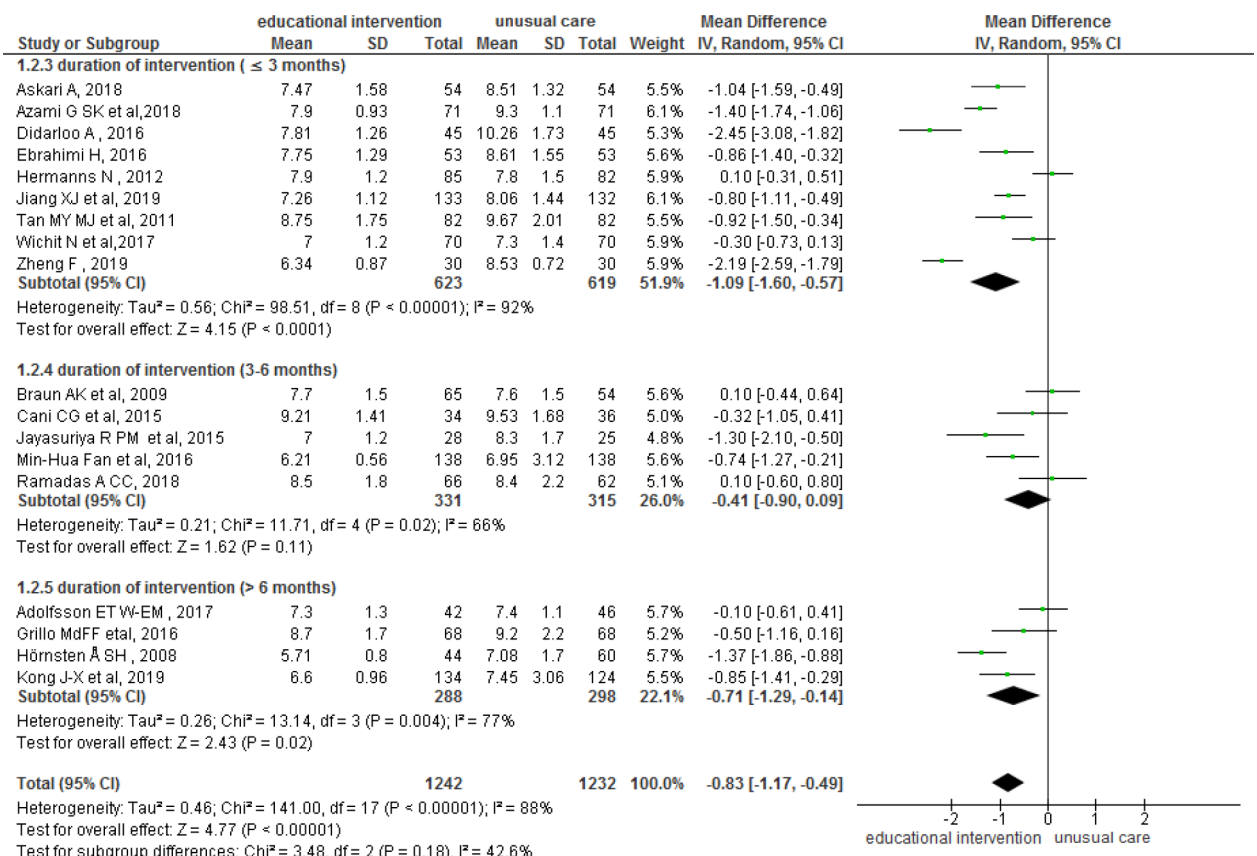

and the true effect may be substantially different from the estimate of the effect size (table 3 ).

\section{DISCUSSION}

Currently, diabetes has emerged as a public health problem that needs effective educational interventions which apply across age, ethnicities and socioeconomic levels. Evidence showed that appropriate self-management education is a vital component of clinical care to improve glycaemic levels and change behavioural outcomes. ${ }^{70}$ In the current meta-analysis, to generate high-quality evidence, only clinical trial studies were included.

This review summarises 19 RCT studies of educational interventions involving 2708 study participants with T2DM that took place in different global regions and health systems. In the present meta-analysis, findings demonstrated that educational intervention has a promising effect on glycaemic control and diabetes knowledge. The finding revealed that educational interventions reduced HbA1c levels by $0.83 \%$ (95\% CI: $1.17 \%$ to $0.49 \%$ ) among patients with T2DM. This finding has a substantial degree of heterogeneity $\left(\mathrm{I}^{2}=88 \%\right)$ indicating variation between included studies. However, there was a slight reduction of MD after sensitivity analysis, $0.70 \%$ (95\% CI: $0.96 \%$ to $0.44 \%$ ), with a moderate degree of heterogeneity $\left(\mathrm{I}^{2}=73 \%\right)$. Our findings are supported by previous meta-analyses, which reported that behavioural and self-management education have a significant benefit in the reduction of $\mathrm{HbAlc}$ levels in patients with diabetes. $^{28} 3738$

The improvement in glycaemic levels is considered to be clinically essential. The UK Diabetes Study revealed

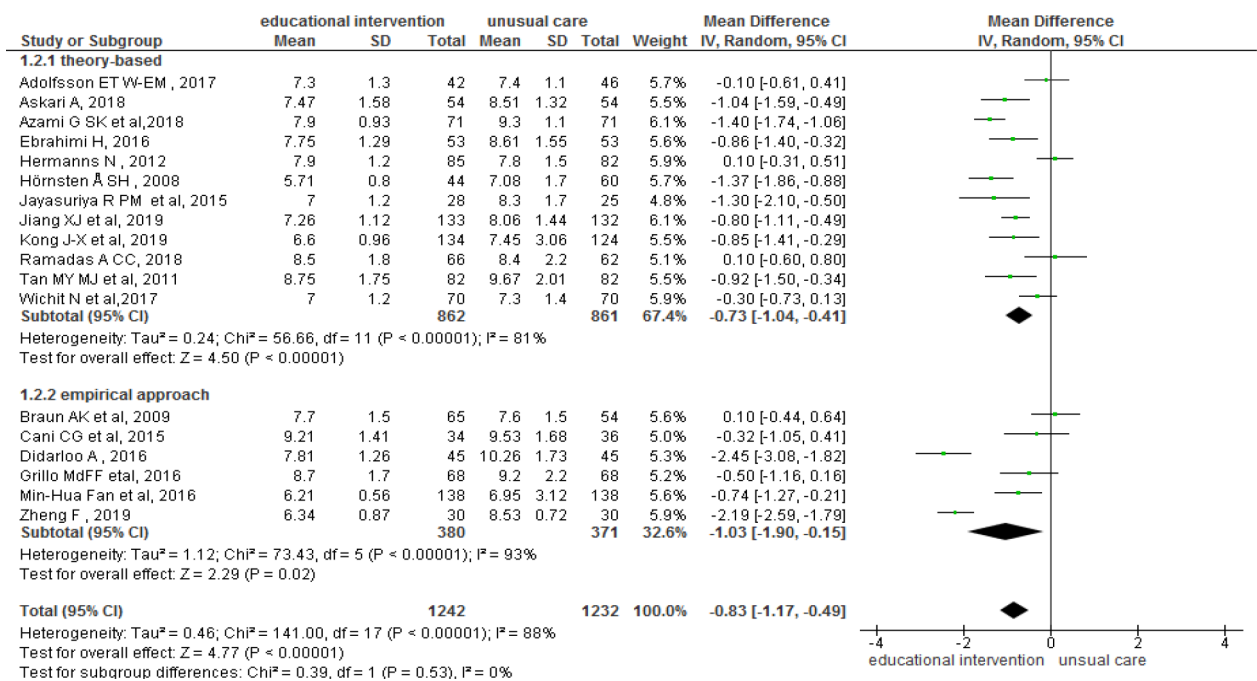

Figure 6 Subgroup analysis based on intervention design. 


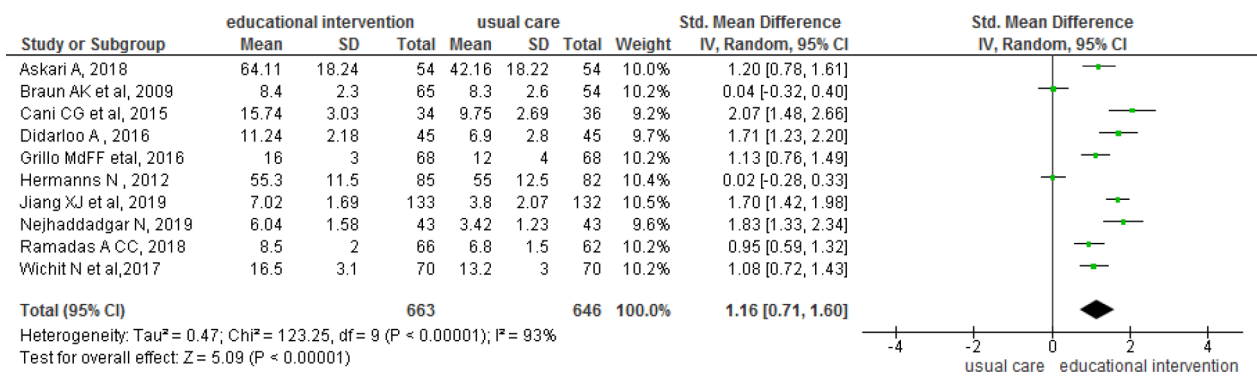

Figure 7 The pooled effect of education interventions on disease knowledge in patients with type 2 diabetes.

that with each $1 \%$ reduction in HbA1c, there is a likelihood of reducing the risk of diabetes complications by $21 \%{ }^{71}$ Similarly, a previous study showed that achieving optimal glycaemic level is likely to reduce the risk of deaths from diabetes complications, such as cardiovascular and cerebrovascular problems. ${ }^{72}$ Moreover, the ADA recognises that diabetes self-management has a vital role in improving glycaemic levels and reducing diabetesrelated complications. ${ }^{73}$

In the current meta-analysis, a subgroup analysis was conducted based on the duration of the educational interventions. Concerning duration of interventions, there was a variation between $<3$ months, $3-6$ months and $>6$ months in the reduction of HbAlc levels. In this metaanalysis, the pooled effect size for short educational interventions (duration $\leq 3$ months) was better than the effect size of longer interventions (duration 3-6months and $>6$ months), -1.09 (95\% CI: -1.60 to $-0.57, \mathrm{p}<0.001)$. One possible explanation may be associated with the initial motivation of the participant to be empowered to obtain positive results in a short period. ${ }^{74}$ In contrast, previous studies reported that longer duration of interventions was more likely related to a significant reduction in HbAlc levels. $^{28} 7576$ Similarly, a meta-analysis study showed that more contact hours were associated with a reduction of HbA1c level. ${ }^{33}$ Moreover, evidence also supported that the duration of contact hours between trainer and patient has a substantial impact on HbA1c levels. ${ }^{18}$ The current findings reflect that the duration of intervention would influence the effectiveness of the educational

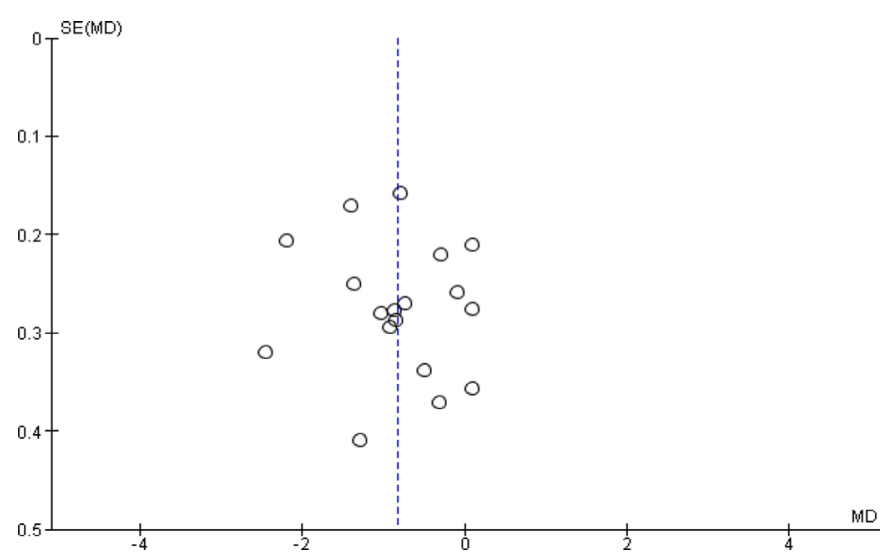

Figure 8 Funnel plot for $\mathrm{HbA} 1 \mathrm{c}$ results. $\mathrm{HbA} 1 \mathrm{c}$, glycosylated haemoglobin; MD, mean difference. intervention among patients with T2DM. Therefore, this disparity should be considered when developing future educational interventions.

In the present review, a subgroup analysis was conducted based on intervention design (theory-based vs empirical educational). Our study indicated that educational interventions benefited all patients regardless of the intervention design. In the current findings, empirical educational intervention showed improvement in glycaemic control level, -1.03 (95\% CI: -1.90 to $-0.15, \mathrm{p}<0.001)$. Similarly, evidence showed that interactive self-management interventions through evidence-based approaches and structured curricula are crucial to improve glycaemic control and behavioural outcomes. ${ }^{77}$ However, another review indicates that in patients with T2DM, theory-based self-management educational interventions improved HbA1c. ${ }^{36}$ Although one-third of the included studies used an empirical approach in designing interventions, and favourable results on glycaemic control were obtained, their specific role in educational interventions has been debated.

In this review, diabetes knowledge showed a significantly higher standardised mean score of correct knowledge of diabetes among the intervention group as compared with the standard care group $(\mathrm{SMD}=1.16$; 95\% CI: 0.71 to $1.60, \mathrm{p}<0.001)$. Similarly, educational interventions were associated with significant improvements in knowledge of diabetes being reported in the previous meta-analyses. ${ }^{78}$ Moreover, there is evidence that education improves knowledge and subsequently promotes behavioural changes among patients with endstage renal disease. ${ }^{80}$ Though significant changes were observed in diabetes knowledge, this finding should be interpreted with caution due to the significant degree of heterogeneity among included studies.

\section{Limitations}

Our study has some limitations that need to be considered in the future. First, studies published in the English language were only considered for this systematic review. Second, there was variation in the included studies in terms of healthcare providers, component of interventions, outcome measures and intervention methods. Third, global representativeness must be considered as it was not possible to identify evidence from all countries of the globe. Fourth, although all the included studies were 


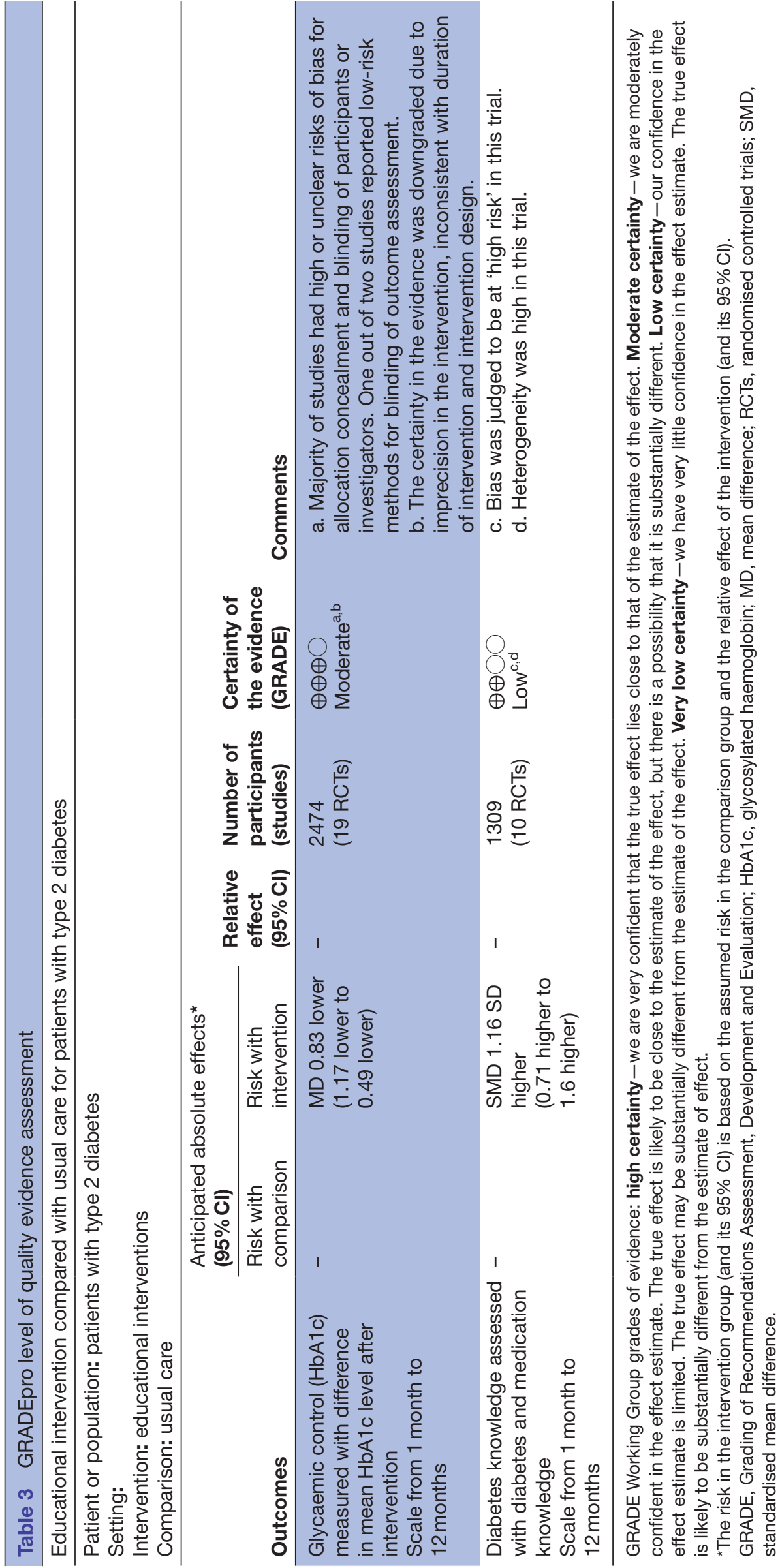

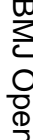

言

등 
randomised controlled trials, some trials had biases, such as lack of allocation concealment, blinding and intentionto-treat analysis.

\section{CONCLUSION}

This systematic review adds to the body of knowledge that suggests that structured diabetes self-management education and support contribute to improving glycaemic outcomes and diabetes knowledge. Therefore, clinicians could make an effort to provide such care to ensure glycaemic control and to improve knowledge of T2DM. Further research is needed to determine the clinical significance of these improvements and their cost-effectiveness.

\section{Implications for practice}

Overall, these data revealed that educational interventions provide a basic benchmark to reduce glycaemic levels and to improve knowledge of T2DM. Importantly, to implement a successful education intervention, it is necessary to consider the duration of intervention and intervention design (empirical education is more effective) in patients with T2DM. Therefore, clinicians should use educational interventions to improve glycaemic control and diabetes knowledge among patients with T2DM. However, before making a practice decision based on the current review, further information from other reviews considering how the role of educational intervention reduced glycaemic level and improved diabetes knowledge should be taken into account. Hence, the certainty of this evidence is not adequate to conclude that interventions will be effective among patients with T2DM.

\section{Implications for research}

Further research is likely to change the estimated effect size of educational interventions in glycaemic control and knowledge of patients with T2DM. Knowledge of diabetes was assessed using different tools, outcome data were measured in heterogeneous ways. Based on this review, future studies of educational interventions would increase our certainty of evidence whether these interventions improve knowledge of diabetes or not by overcoming limitations of existing studies. Therefore, future educational intervention studies should be designed to evaluate individual-centred outcomes and become new priorities to support in clinical decision-making.

\section{Author affiliations \\ ${ }^{1}$ Nursing, Debre Berhan University, Debre Berhan, Ethiopia \\ ${ }^{2}$ Nursing, Debre Markos University, Debre Markos, Amhara, Ethiopia \\ ${ }^{3}$ Midwifery, Debre Markos University, Debre Markos, Amhara, Ethiopia \\ ${ }^{4}$ Nursing, Woldia University, Woldia, Amhara, Ethiopia \\ ${ }^{5}$ Nursing, University of Saskatchewan, Saskatoon, Saskatchewan, Canada \\ ${ }^{6}$ Pediatric and Child Health, Debre Berhan University, Debre Berhan, Amhara, Ethiopia}

Contributors WSS, YAA and TY developed the protocol and were involved in the design, selection of study, data extraction, statistical analysis and developing the initial drafts of the manuscript. AMK, WSS, PMP and MD were involved in data extraction, quality assessment, statistical analysis and revision. WSS and YAA prepared and edited the final draft of the manuscript. All authors read and approved the final draft of the manuscript. WSS has taken the full responsibility for the work and/or the conduct of the study, had access to the data and controlled the decision to publish.

Funding The authors have not declared a specific grant for this research from any funding agency in the public, commercial or not-for-profit sectors.

Competing interests None declared.

Patient and public involvement Patients and/or the public were not involved in the design, or conduct, or reporting, or dissemination plans of this research.

Patient consent for publication Not required.

Provenance and peer review Not commissioned; externally peer reviewed.

Data availability statement All data relevant to the study are included in the article or uploaded as supplemental information. All relevant data are within the paper and supporting information files. There is no separate data set to share.

Open access This is an open access article distributed in accordance with the Creative Commons Attribution Non Commercial (CC BY-NC 4.0) license, which permits others to distribute, remix, adapt, build upon this work non-commercially, and license their derivative works on different terms, provided the original work is properly cited, appropriate credit is given, any changes made indicated, and the use is non-commercial. See: http://creativecommons.org/licenses/by-nc/4.0/.

\section{ORCID iDs}

Wondimeneh Shibabaw Shiferaw http://orcid.org/0000-0003-4348-591X

Tadesse Yirga Akalu http://orcid.org/0000-0002-5475-6183

Ayelign Mengesha Kassie http://orcid.org/0000-0003-1505-9390

Yared Asmare Aynalem http://orcid.org/0000-0002-3005-2296

\section{REFERENCES}

1 Fan W. Epidemiology in diabetes mellitus and cardiovascular disease. Cardiovasc Endocrinol 2017;6:8-16.

2 Roglc G, Varghese C, Cowan M. Global report on diabetes (World Health Organization). WHO Library Cataloguing-in-publication Data, 2016.

3 Cho $\mathrm{NH}$, Shaw JE, Karuranga S, et al. IDF diabetes atlas: global estimates of diabetes prevalence for 2017 and projections for 2045. Diabetes Res Clin Pract 2018;138:271-81.

4 International diabetes federation. IDF diabetes atlas teB, Belgium. International Diabetes Federation, 2015.

5 Zheng Y, Ley SH, Hu FB. Global aetiology and epidemiology of type 2 diabetes mellitus and its complications. Nat Rev Endocrinol 2018;14:88-98.

6 Lyles CR, Grothaus L, Reid RJ, et al. Communication about diabetes risk factors during between-visit encounters. Am J Manag Care 2012;18:807-15.

7 Meng X-H, Huang Y-X, Rao D-P, et al. Comparison of three data mining models for predicting diabetes or prediabetes by risk factors. Kaohsiung J Med Sci 2013;29:93-9.

8 World Health Organization. Global report on diabetes, 2016.

9 American Diabetes Association. 6. Glycemic targets: standards of medical care in diabetes-2020. Diabetes Care 2020;43:S66-76.

10 Gibbons $\mathrm{CH}$, Goebel-Fabbri A. Microvascular complications associated with rapid improvements in glycemic control in diabetes. Curr Diab Rep 2017:17:48.

11 Haghighatpanah M, Nejad ASM, Haghighatpanah M, et al. Factors that correlate with poor glycemic control in type 2 diabetes mellitus patients with complications. Osong Public Health Res Perspect 2018;9:167-74

12 Dutta T, Kudva YC, Persson X-MT, et al. Impact of long-term poor and good glycemic control on metabolomics alterations in type 1 diabetic people. J Clin Endocrinol Metab 2016;101:1023-33.

13 Lau CY, Qureshi AK, Scott SG. Association between glycaemic control and quality of life in diabetes mellitus. J Postgrad Med 2004;50:189-93.

14 Degli Esposti L, Saragoni S, Buda S, et al. Glycemic control and diabetes-related health care costs in type 2 diabetes; retrospective analysis based on clinical and administrative databases. Clinicoecon Outcomes Res 2013;5:193-201.

15 Control Group, Turnbull FM, Abraira C, et al. Intensive glucose control and macrovascular outcomes in type 2 diabetes. Diabetologia 2009;52:2288-98. 
16 Hemmingsen B, Lund SS, Gluud C, et al. Intensive glycaemic control for patients with type 2 diabetes: systematic review with metaanalysis and trial sequential analysis of randomised clinical trials. BMJ 2011;343:d6898.

17 Sharifirad G, Najimi A, Hassanzadeh A, et al. Application of BASNEF educational model for nutritional education among elderly patients with type 2 diabetes: improving the glycemic control. J Res Med Sci 2011;16:1149-58.

18 Norris SL, Lau J, Smith SJ, et al. Self-Management education for adults with type 2 diabetes: a meta-analysis of the effect on glycemic control. Diabetes Care 2002;25:1159-71.

19 Ellis SE, Speroff T, Dittus RS, et al. Diabetes patient education: a meta-analysis and meta-regression. Patient Educ Couns 2004;52:97-105.

20 Deakin TA MC, Cade JE, Williams R. Group based training for self-management strategies in people with type 2 diabetes mellitus. Cochrane database of systematic reviews 2005;18:CD003417.

21 Soundarya M, Asha A, Mohan V. Role of a diabetes educator in the management of diabetes. Int J Diabetes Dev Ctries 2004;24:65-8.

22 Berikai P, Meyer PM, Kazlauskaite R, et al. Gain in patients knowledge of diabetes management targets is associated with better glycemic control. Diabetes Care 2007;30:1587-9.

23 Hartz A, Kent S, James $\mathrm{P}$, et al. Factors that influence improvement for patients with poorly controlled type 2 diabetes. Diabetes Res Clin Pract 2006;74:227-32.

24 Rhee MK, Slocum W, Ziemer DC, et al. Patient adherence improves glycemic control. Diabetes Educ 2005;31:240-50.

25 Hussain Z, Alkharaiji M, Idris I. Evaluating the effect of inpatient diabetes education on length of stay, readmission rates and mortality rates: a systematic review. Br J Diabetes 2020;20:96-103.

26 Healy SJ, Black D, Harris C, et al. Inpatient diabetes education is associated with less frequent Hospital readmission among patients with poor glycemic control. Diabetes Care 2013;36:2960-7.

27 Bukhsh A, Khan TM, Sarfraz Nawaz M, et al. Association of diabetes knowledge with glycemic control and self-care practices among Pakistani people with type 2 diabetes mellitus. Diabetes Metab Syndr Obes 2019;12:1409-17

28 Azami G, Soh KL, Sazlina S-G, et al. Behavioral interventions to improve self-management in Iranian adults with type 2 diabetes: a systematic review and meta-analysis. J Diabetes Metab Disord 2018;17:365-80.

29 Powers MA, Bardsley J, Cypress M, et al. Diabetes self-management education and support in type 2 diabetes: a joint position statement of the American diabetes association, the American association of diabetes educators, and the Academy of nutrition and dietetics. The Diabetes Educ 2017;43:40-53.

30 Powers MA, Bardsley JK, Cypress M, et al. Diabetes selfmanagement education and support in adults with type 2 diabetes: a consensus report of the American Diabetes Association, the Association of Diabetes Care \& Education Specialists, the Academy of Nutrition and Dietetics, the American Academy of Family Physicians, the American Academy of PAs, the American Association of Nurse Practitioners, and the American Pharmacists Association. Sci Diabetes Self Manag Care 2021;47:54-73.

31 Bos M, Agyemang C. Prevalence and complications of diabetes mellitus in northern Africa, a systematic review. BMC Public Health 2013;13:387.

32 Somannavar S, Lanthorn H, Deepa M. Increased awareness about diabetes and its complications in a whole city: Effectiveness of the "prevention, awareness, counselling and evaluation"[PACE] Diabetes Project [PACE-6]. J Assoc Physicians India 2008;56:495-502.

33 Chrvala CA, Sherr D, Lipman RD. Diabetes self-management education for adults with type 2 diabetes mellitus: a systematic review of the effect on glycemic control. Patient Educ Couns 2016;99:926-43.

34 Schapira MM, Swartz S, Ganschow PS, et al. Tailoring educational and behavioral interventions to level of health literacy: a systematic review. MDM Policy Pract 2017;2:238146831771447-74.

35 Murugesan N, Snehalatha C, Shobhana R, et al. Awareness about diabetes and its complications in the general and diabetic population in a City in southern India. Diabetes Res Clin Pract 2007;77:433-7.

36 Zhao F-F, Suhonen R, Koskinen S, et al. Theory-based selfmanagement educational interventions on patients with type 2 diabetes: a systematic review and meta-analysis of randomized controlled trials. J Adv Nurs 2017;73:812-33.

37 Hildebrand JA, Billimek J, Lee J-A, et al. Effect of diabetes selfmanagement education on glycemic control in Latino adults with type 2 diabetes: a systematic review and meta-analysis. Patient Educ Couns 2020;103:266-75.

38 Aquino JA, Baldoni NR, Flôr CR, et al. Effectiveness of individual strategies for the empowerment of patients with diabetes mellitus: a systematic review with meta-analysis. Prim Care Diabetes 2018;12:97-110.

39 Moher D, Shamseer L, Clarke M, et al. Preferred reporting items for systematic review and meta-analysis protocols (PRISMA-P) 2015 statement. Syst Rev 2015;4:1.

40 McGowan J, Sampson M, Salzwedel DM, et al. PRESS peer review of electronic search strategies: 2015 guideline statement. J Clin Epidemiol 2016;75:40-6.

41 Higgins JP. Cochrane Handbook for systematic reviews of interventions version 5.0. 1. The Cochrane Collaboration, 2008. http://www cochrane-handbook org

42 Higgins J, Green S, eds. Cochrane Handbook for Systematic Reviews of Interventions. Version 5.1. 0 [updated March 2011]. The Cochrane Collaboration, 2011.

43 Sterne JAC, Savović J, Page MJ, et al. RoB 2: a revised tool for assessing risk of bias in randomised trials. BMJ 2019;366:14898.

44 Guyatt GH, Oxman AD, Kunz R, et al. What is "quality of evidence" and why is it important to clinicians? BMJ 2008;336:995-8.

45 GRADEpro G. GRADEpro guideline development tool [software], 2015. McMaster University

46 Borenstein M, Hedges LV, Higgins JPT, et al. A basic introduction to fixed-effect and random-effects models for meta-analysis. Res Synth Methods 2010;1:97-111.

47 Higgins JPT, Thompson SG, Deeks JJ, et al. Measuring inconsistency in meta-analyses. BMJ 2003;327:557-60.

48 Cohen J. Statistical power analysis for the behavioral sciences. Academic press, 2013

49 Egger M, Smith GD, Schneider M. Bias in meta-analysis detected by a simple, graphical test. increase in studies of publication bias coincided with increasing use of meta-analysis. BMJ 1997;316:629-34.

50 Sterne JAC, Sutton AJ, loannidis JPA, et al. Recommendations for examining and interpreting funnel plot asymmetry in meta-analyses of randomised controlled trials. BMJ 2011;343:d4002.

51 Cani CG, Lopes LdaSG, Queiroz M, et al. Improvement in medication adherence and self-management of diabetes with a clinical pharmacy program: a randomized controlled trial in patients with type 2 diabetes undergoing insulin therapy at a teaching hospital. Clinics 2015;70:102-6.

52 MdFF G, Neumann CR, Scain SF, et al. Diabetes education in primary care: a randomized clinical trial. Cadernos de Saude Publica 2016;32:e00097115.

53 Zheng F, Liu S, Liu Y, et al. Effects of an outpatient diabetes selfmanagement education on patients with type 2 diabetes in China: a randomized controlled trial. J Diabetes Res 2019;2019:1-7.

54 Jiang $\mathrm{X}$-J, Jiang $\mathrm{H}$, Lu Y-H, et al. The effectiveness of a self-efficacyfocused structured education programme on adults with type 2 diabetes: a multicentre randomised controlled trial. J Clin Nurs 2019;28:3299-309.

55 Kong J-X, Zhu L, Wang H-M, et al. Effectiveness of the chronic care model in type 2 diabetes management in a community health service center in China: a group randomized experimental study. J Diabetes Res 2019;2019:1-12.

56 Hermanns N, Kulzer B, Maier B, et al. The effect of an education programme (MEDIAS 2 ICT) involving intensive insulin treatment for people with type 2 diabetes. Patient Educ Couns 2012;86:226-32.

57 Braun AK, Kubiak T, Kuntsche J, et al. SGS: a structured treatment and teaching programme for older patients with diabetes mellitus--a prospective randomised controlled multi-centre trial. Age Ageing 2009;38:390-6

58 Didarloo A, Shojaeizadeh D, Alizadeh M. Impact of educational intervention based on interactive approaches on beliefs, behavior, hemoglobin A1c, and quality of life in diabetic women. Int J Prev Med 2016;7:38.

59 Askari A, Jeihooni AK, Kashfi SM, et al. The effect of educational program based on belief, attitude, subjective norm, and enabling factors model on changing the metabolic indices in elderly patients with type II diabetes. Int J Prev Med 2018;9:74

60 Azami G, Soh KL, Sazlina SG, et al. Effect of a nurse-led diabetes self-management education program on glycosylated hemoglobin among adults with type 2 diabetes. J Diabetes Res 2018;2018:1-12.

61 Ebrahimi H, Sadeghi M, Amanpour F, et al. Evaluation of empowerment model on indicators of metabolic control in patients with type 2 diabetes, a randomized clinical trial study. Prim Care Diabetes 2016;10:129-35.

62 Nejhaddadgar N, Darabi F, Rohban A, et al. The effectiveness of self-management program for people with type 2 diabetes mellitus based on PRECEDE-PROCEDE model. Diabetes Metab Syndr 2019;13:440-3

63 Tan MY, Magarey JM, Chee SS, et al. A brief structured education programme enhances self-care practices and improves glycaemic 
control in Malaysians with poorly controlled diabetes. Health Educ Res 2011;26:896-907.

64 Ramadas A, Chan CKY, Oldenburg B, et al. Randomised-controlled trial of a web-based dietary intervention for patients with type 2 diabetes: changes in health cognitions and glycemic control. BMC Public Health 2018;18:716.

65 Hörnsten A, Stenlund H, Lundman B, et al. Improvements in HbA1c remain after 5 years--a follow up of an educational intervention focusing on patients' personal understandings of type 2 diabetes. Diabetes Res Clin Pract 2008;81:50-5.

66 Adolfsson ET, Walker-Engström M-L, Smide B, et al. Patient education in type 2 diabetes: a randomized controlled 1-year followup study. Diabetes Res Clin Pract 2007;76:341-50.

67 Wichit N, Mnatzaganian G, Courtney M, et al. Randomized controlled trial of a family-oriented self-management program to improve selfefficacy, glycemic control and quality of life among Thai individuals with type 2 diabetes. Diabetes Res Clin Pract 2017;123:37-48.

68 Jayasuriya R, Pinidiyapathirage MJ, Jayawardena R, et al. Translational research for diabetes self-management in Sri Lanka: a randomized controlled trial. Prim Care Diabetes 2015;9:338-45.

69 Fan M-H, Huang B-T, Tang Y-C, et al. Effect of individualized diabetes education for type 2 diabetes mellitus: a single-center randomized clinical trial. Afr Health Sci 2016:16:1157-62.

70 American Diabetes Association. Standards of Medical Care in Diabetes-2019 Abridged for Primary Care Providers. Clin Diabetes 2019;37:11-34.

71 Stratton IM, Adler Al, Neil HA, et al. Association of glycaemia with macrovascular and microvascular complications of type 2 diabetes (UKPDS 35): prospective observational study. BMJ 2000;321:405-12.
72 Association AD. Implications of the diabetes control and complications trial. Diabetes Care 2002;25:S25-7.

73 American Diabetes Association. (4) foundations of care: education, nutrition, physical activity, smoking cessation, psychosocial care, and immunization. Diabetes Care 2015;38 Suppl:S20-30.

74 Funnell MM, Anderson RM. Empowerment and self-management of diabetes. Clin Diabetes 2004;22:123-7.

75 McEwen MM, Pasvogel A, Gallegos G, et al. Type 2 diabetes selfmanagement social support intervention at the U.S.-Mexico border. Public Health Nurs 2010;27:310-9.

76 Glazier RH, Bajcar J, Kennie NR, et al. A systematic review of interventions to improve diabetes care in socially disadvantaged populations. Diabetes Care 2006;29:1675-88.

77 Rickheim PL, Weaver TW, Flader JL, et al. Assessment of group versus individual diabetes education: a randomized study. Diabetes Care 2002;25:269-74.

78 Creamer J, Attridge M, Ramsden M, et al. Culturally appropriate health education for type 2 diabetes in ethnic minority groups: an updated cochrane review of randomized controlled trials. Diabet Med 2016;33:169-83.

79 Cheng L, Sit JWH, Choi K-C, et al. Effectiveness of interactive selfmanagement interventions in individuals with poorly controlled type 2 diabetes: a meta-analysis of randomized controlled trials. Worldviews Evid Based Nurs 2017;14:65-73.

80 Tsay S-L, Hung L-O. Empowerment of patients with end-stage renal disease--a randomized controlled trial. Int J Nurs Stud 2004;41:59-65. 